\title{
Der Krebspatient in der Anästhesie
}

\author{
Eine neue Patientengruppe mit neuen Anforderungen
}

Die Fortschritte der modernen Onkologie mit ihren differenzierten Behandlungsmöglichkeiten haben auch beträchtliche Auswirkungen auf die anästhesiologische, intensiv- und notfallmedizinische Betreuung von Tumorpatienten. Als auffälligste Entwicklung sehen Alt-Epping et al. die Vielzahl neu zugelassener Substanzen, „die ihre antitumorale Wirkung weniger aufgrund konventioneller zytotoxischer Wirkmechanismen wie Signaltransduktionshemmung oder Apoptoseregulation entfalten und die heute mit dem Sammelbegriff der ,targeted therapies' bezeichnet werden."

Das zu bisher eingesetzten Zytostatika unterschiedliche Nebenwirkungsspektrum der neuen Substanzen, sowie die grundsätzlich eher zur Tumorstabilisierung denn Tumorreduktion tendierende Wirkung stelle die Anästhesiologie vor neue Herausforderungen hinsichtlich Narkoseeinschätzung und perioperativer Führung einer zuvor nichtexistenten Gruppe von Patienten mit Tumorerkrankungen, stellen die Autoren fest.

Es bedarf daher einer zunehmend genaueren Differenzierung des zugrundeliegenden onkologischen Therapiekonzepts, der applizierten Substanzgruppen und ihrer pharmakologischen Eigenschaften und Nebenwirkungen. So sind, laut Alt-Epping et al., aus infektiologischer Perspektive die verschiedenen krankheitsassoziierten oder medikamentös induzierten Immundefekt- syndrome mit einer notwendigen Berücksichtigung atypischer, viraler oder Pilzerreger bei der empirischen Antibiotikaauswahl von Interesse. Nebenwirkungen und Arzneimittelinteraktionen nehmen bei der Verabreichung von Tumortherapeutika einen hohen Stellenwert ein, betonen die Autoren, wobei sich das Cytochrom P450 System als

\section{Zusätzlich zu neuen Substan- zen stellen unterschiedliche multimodale Konzepte neue Situationen für die Anästhesie und Intensivmedizin dar.}

Schnittstelle für Arzneimittelinteraktionen erweise. Von den bekannten Hemmstoffen des Cytochrom P450 Systems kommen in der Tumortherapie vor allem Allopurinol, Ciprofloxacin, Makrolidantibiotika, Azolanimykotika, östrogenhaltige Präparate oder Interferon- $\alpha$ zum Einsatz. Die zunehmende Zahl neuer Substanzen stellt in der Anästhesie und Intensivmedizin eine zusätzliche Herausforderung dar.

Darüberhinaus stellen unterschiedliche multimodale Konzepte neue Situationen für die Anästhesie und Intensivmedizin dar. Beim nicht-kleinzelligen Bronchuskarzinom wurde beispielsweise eine postoperative Mortalitätsrate von 26 Prozent nach
Pneumektomie nach vorangegangener neoadjuvanter Radiochemotherapie meist aufgrund respiratorischer Komplikationen/ ARDS aufgezeigt.

Die unterschiedlichen Therapiekonzepte wie beispielsweise die metronomische Erhaltungstherapie, die bestimmte solide Tumoren zu einer „chronischen“ Erkrankung gemacht haben, und damit die Wahrscheinlichkeit, dass diese Patienten unter laufender Tumortherapie eine Allgemeinanästhesie benötigen erhöht haben, erfordern vom Anästhesisten und Intensivmediziner besondere Aufmerksamkeit. Hinzu kommt die demographische Entwicklung, die auch eine Zunahme der in höherem Alter auftretenden und damit häufiger werdenden Tumore bewirkt und in der Folge onkologische Patienten in den Wirkungsbereich der Anästhesie und Intensivmedizin führt. Auf der ethischen Ebene bedarf es, so die Autoren, eines hohen Maßes an kommunikativer Kompetenz und klinischer Abwägung. „Pharmakotherapeutische, konzeptuelle, epidemiologische und ethische Aspekte der modernen Onkologie werden auch konkrete Auswirkungen auf die Anästhesie, Intensiv- und Notfallmedizin haben und neue Bewertungsgrundlagen für neue klinische Konstellationen von Tumorpatienten erfordern", schließen die Autoren.

B. Alt-Epping, S. Alt-Epping, M. Quintel, F. Nauck: Entwicklungen der modernen Onkologie. Auswirkungen auf Anästhesie und Intensivmedizin, In: Der Anästhesist 2009

\section{IMPRESSUM}

Herausgeber und Verleger: Springer-Verlag GmbH, Professional Media, Sachsenplatz 4-6, 1201 Wien, Austria; Tel.: 01/330 24 15-0, Fax: 01/330 24 26-260; Internet: www.springer.at; www.SpringerMedizin.at; Geschäftsführung: Mag. Katharina Oppitz; Leitung: Mag. Margarete Zupan; Redaktion: Verantwortlicher Redakteur: Dr. Verena Kienast; Mitarbeiter dieser Ausgabe: Dr. Renate Höhl, Mag. Patricia Herzberger, Raoul Mazhar, Mag. Tanja Fabsits; Redaktionssekretariat: Susanna Hinterberger; Produktion: Mag. (FH) Dorothea Woliński; Anzeigen: Dipl. Tzt. Elise Haidenthaller; Es gilt die Anzeigenpreisliste 2010; Erscheinungsweise: $6 x$ jährlich; Abonnements: Bezugspreis: 1 Jahr Euro 43,- exkl. MwSt; Verlagsort: Wien; Herstellungsort: Wien; Erscheinungsort: Wien; P.b.b./Verlagspostamt: 1201 Wien; ISSN 1869-1757; ISSN Electronic 1613-7817; Graphik: Ursula Szorger; Layout: Wojtek Grzymala; Druck: Holzhausen Druck \& Medien GmbH, 1140 Wien-Auhof, Holzhausenplatz 1.

Alle namentlich gekennzeichneten Beiträge spiegeln nicht unbedingt die Meinung der Redaktion wider. Diese Beiträge fallen somit in den persönlichen Verantwortungsbereich des Verfassers. Die Redaktion übernimmt keine Haftung für unaufgefordert eingesandte Manuskripte. Mit „Advertorial“ gekennzeichnete Beiträge sind bezahlte Einschaltungen. Urheberrecht: Mit der Annahme eines Beitrags zur Veröffentlichung erwirbt der Verlag vom Autor alle Rechte, insbesondere das Recht der weiteren Vervielfältigung zu gewerblichen Zwecken mit Hilfe photomechanischer oder anderer Verfahren. Die Zeitschrift sowie alle in ihr enthaltenen einzelnen Beiträge und Abbildungen sind urheberrechtlich geschützt. Jede Verwertung, die nicht ausdrücklich vom Urheberrechtsgesetz zugelassen ist, bedarf der vorherigen schriftlichen Zustimmung des Verlags. Das gilt insbesondere für Vervielfältigungen, Bearbeitungen, Übersetzungen, Mikroverfilmungen und die Einspeicherung und Verarbeitung in elektronischen Systemen. Auch die Rechte der Wiedergabe durch Vortrag. Funk- und Fernsehsendungen, im Magnettonverfahren oder auf ähnlichem Wege bleiben nicht zu der Annahme, dass solche Namen im Sinne der Warenzeichen- und Markenschutz-Gesetzgebung als frei zu betrachten wären und daher von jedermann benütz werden dürfen. Für Angaben über Dosierungsanweisungen und Applikationsformen kann vom Verlag keine Gewähr übernommen werden. Derartige Angaben müssen vom jeweiligen Anwender im Einzelfall anhand Literaturstellen auf ihre Richtigkeit überprüft werden.

Eigentümer und Copyright-Inhaber: ㅇ 2010 Springer-Verlag/Wien. SpringerWienNewYork ist ein Unternehmen von Springer Science+Business Media; Beilage zur wiener klinischen wochenschrift 3-4/2010

Offenlegung gem. § 25 Abs. 1 bis 3 Mediengesetz: Unternehmensgegenstand: Verlag von wissenschaftlichen Büchern und Zeitschriften. An der Springer-Verlag GmbH ist beteiligt: Springer Austria GmbH, Sachsenplatz 4-6, 1201 Wien, Austria, zu 99,8 \%. Geschäftsführung: Mag. Katharina Oppitz, Sachsenplatz 4-6, 1201 Wien, Austria. 